ESAIM: PROCEEDINGS, March 2012, Vol. 35, p. 114-121

Fédération Denis Poisson (Orléans-Tours) et E. Trélat (UPMC), Editors

\title{
STABILITÉ SOUS CONDITION CFL NON LINÉAIRE
}

\author{
ERWAN DERIAZ ${ }^{1}$ AND DMITRY KOLOMENSKIY ${ }^{2}$
}

\begin{abstract}
We present a basic althought little known numerical stability condition: for convection equations, the von Neumann stability constraint $\left\|u_{n+1}\right\|_{L^{2}} \leq(1+C \Delta t)\left\|u_{n}\right\|_{L^{2}}$ drives to the stability condition $\Delta t \leq C \Delta x^{\alpha}$ with $\alpha=\frac{p(2 q-1)}{q(2 p-1)}$ where $p$ is an integer linked to the stability domain of the time scheme and $q \geq p$ an integer linked to the upwind property of the space discretization (in the centered case we have $q=+\infty$ and $\left.\alpha=\frac{2 p}{2 p-1}\right)$.

Résumé. Nous présentons une étude de stabilité aux conclusions originales : pour les équations de convection, la contrainte $\left\|u_{n+1}\right\|_{L^{2}} \leq(1+C \Delta t)\left\|u_{n}\right\|_{L^{2}}$ fait apparaître la condition de stabilité $\Delta t \leq C \Delta x^{\alpha}$ avec $\alpha=\frac{p(2 q-1)}{q(2 p-1)}$ où $p$ est un entier lié au domaine de stabilité du schéma en temps et $q \geq p$ un autre entier lié au caractère upwind de la discrétisation en espace (dans le cas centré, $q=+\infty)$. Des exemples numériques montrent la pertinence de cette étude.
\end{abstract}

\section{INTRODUCTION}

Lorsqu'on considère la solution d'une équation aux dérivées partielles $\left(u_{n}\right)$ discrétisée uniformément en espace et en temps, la plupart des études de stabilité négligent le terme en $O(\Delta t)$ dans l'inégalité $\left\|u_{n+1}\right\|_{L^{2}} \leq(1+$ $O(\Delta t))\left\|u_{n}\right\|_{L^{2}}$. Or, contrairement à une idée très répandue [8], autoriser une croissance de la solution en $O(\Delta t)$ par pas de temps affecte considérablement les conditions de stabilité. Certains schémas spectraux d'ordre deux en temps (Runge-Kutta, Adams-Bashforth...), très utilisés en mécanique des fluides se trouvent être instables sous condition CFL linéaire $\Delta t \leq C \Delta x$ alors qu'ils permettent de calculer des solutions satisfaisantes en des temps raisonnables [4]. Une étude de stabilité de von Neumann de ces schémas, avec le critère $\left\|u_{n+1}\right\|_{L^{2}} \leq$ $(1+O(\Delta t))\left\|u_{n}\right\|_{L^{2}}$, mène à la condition de stabilité $\Delta t \leq C \Delta x^{\frac{4}{3}}$.

Il est possible de généraliser cette condition de stabilité, et de construire des schémas en temps stables sous la condition $\Delta t \leq C \Delta x^{\frac{2 p}{2 p-1}}$ avec $p$ un entier. Cet entier $p$ est lié au degré de tangence du domaine de stabilité du schéma en temps dont le bord suit l'équation $x=-K y^{2 p}$, avec $K>0$, à l'axe $(O y)$. Cette condition reste valable pour tous les schémas ayant une discrétisation conservative en espace : schémas spectraux, différences finies centrées, schémas Galerkin. Seul le caractère upwind d'un schéma peut contrecarrer cette instabilité.

L'article suivra le plan suivant : nous présenterons d'abord rapidement les éléments de base que sont les équations hyperboliques, la condition CFL (Courant Friedrichs Lewy) et le principe de l'analyse de stabilité de von Neumann; puis nous ferons apparaître la condition $\Delta t \leq C \Delta x^{\alpha}$ avec $\alpha>1$ que nous testerons numériquement; enfin, en conclusion, nous tenterons d'expliquer pourquoi des schémas d'ordre deux instables sous condition CFL restent très utilisés pour simuler la turbulence malgré une condition plus stricte sur le pas de temps.

${ }^{1}$ Laboratoire de Mécanique, Modélisation \& Procédés Propres - UMR-6181 CNRS - IMT La Jetée, Technopôle de ChâteauGombert, 38 rue Frédéric Joliot-Curie, 13451 Marseille Cedex 13

2 CERFACS, 42, Avenue Gaspard Coriolis 31057 - Toulouse Cedex 01

(C) EDP Sciences, SMAI 2012 


\section{Notions ET PRINCIPES DE BASE}

\subsection{Les équations hyperboliques}

Les conditions de stabilité présentées ci-dessous s'appliquent à beaucoup d'équations aux dérivées partielles. Car, en ce qui concerne la stabilité numérique, celles-ci se ramènent souvent en première approximation, à des équations de transport, par linéarisation ou par factorisation.

On se donne une équation $(E) \partial_{t} u=F(u)$. Et on considère une perturbation de $u$ par une fonction très oscillante $h, u \mapsto u_{\varepsilon}=u+\varepsilon h$ avec $\|h\|<<\left\|\partial_{x} h\right\|$. Si $(E)$ est hyperbolique, alors elle se linéarise en

$$
\partial_{t} h=L(u) \partial_{x} h+o(\varepsilon)+o\left(\left\|\partial_{x} h\right\|\right) .
$$

Par ailleurs, si l'équation hyperbolique $(E)$ implique une dérivée seconde en temps $(E) \partial_{t}^{2} u=F(u)$, elle se met sous la forme $\partial_{t} X=M(X)$ avec $X=\left(u, \partial_{t} u\right)^{\prime}$, et $M$ se linéarise comme en (1). C'est par exemple le cas de l'équation des ondes $\partial_{t}^{2} u=c^{2} \partial_{x}^{2} u$ avec $M(X)=\left(\begin{array}{cc}0 & 1 \\ c^{2} \partial_{x}^{2} & 0\end{array}\right) X$.

Ainsi, une fois introduite l'analyse de stabilité de von Neumann, nous nous cantonnerons à une analyse rigoureuse de la stabilité numérique de l'équation de transport :

$$
\partial_{t} u+a \partial_{x} u=0, \quad \text { avec } u: \mathbb{R}_{+} \times \mathbb{R} \rightarrow \mathbb{R},(t, x) \mapsto u(t, x) .
$$

\subsection{Analyse de stabilité de von Neumann et condition CFL}

Initialement, la condition CFL du nom des trois auteurs de l'article fondateur [1], s'énonçait de la façon suivante : "Le domaine de calcul doit inclure le domaine d'influence physique" et se traduisait par l'inégalité : $a \Delta t \leq \Delta x$. Ce critère était donc physique avant d'être numérique. Cependant, par glissement, la désignation CFL est maintenant passée dans le vocabulaire comme condition suffisante de stabilité liant les pas de temps $\Delta t$ et d'espace $\Delta x$. Ce critère de stabilité exact pour l'équation de transport peut s'obtenir par une analyse de stabilité de von Neumann.

Le cadre de l'analyse de stabilité de von Neumann se restreint à des équations linéaires à coefficients constants sur un domaine infini ou périodique : $(E) \partial_{t} u=F u$. Elle consiste à isoler une fréquence d'oscillation $\xi$ de $u(x)=\frac{1}{2 \pi} \int_{\mathbb{R}} u_{\xi}(t, x) d \xi$ avec $u_{\xi}(t, x)=\hat{u}(t, \xi) e^{i \xi x}$. Si on note alors $\sigma(\xi)$ le symbole de l'opérateur $F$ : $\widehat{F u}(t, \xi)=\sigma(\xi) \hat{u}(t, \xi)^{1}$, l'équation $(E)$ se ramène à une équation différentielle ordinaire : $\partial_{t} \hat{u}=\sigma \hat{u}$ avec $\sigma \in \mathbb{C}$. Dans le cas de l'équation de transport (2), $\sigma=-i a \xi \in i \mathbb{R}$.

Considérons maintenant une solution $u$ de $(E)$ sur $[0, T]$ et sa discrétisée en temps $u_{n}$ prise aux instants $n \Delta t$. Un schéma de type Runge-Kutta se construit en utilisant des pas de temps intermédiaires. Par exemple on obtient un schéma de Runge-Kutta d'ordre deux en prenant :

$$
u_{n+1}=u_{n}+\Delta t F\left(u_{n}+\frac{\Delta t}{2} F u_{n}\right)=u_{n}+\Delta t F u_{n}+\frac{\Delta t^{2}}{2} F^{2} u_{n} .
$$

Si on applique la transformée de Fourier en espace à l'équation (3), on obtient :

$$
\widehat{u_{n+1}}=\widehat{u_{n}}+\Delta t \sigma \widehat{u_{n}}+\frac{\Delta t^{2}}{2} \sigma^{2} \widehat{u_{n}}=\left(1+\Delta t \sigma+\frac{\Delta t^{2}}{2} \sigma^{2}\right) \widehat{u_{n}}
$$

Ainsi, en prenant $z=\Delta t \sigma \in \mathbb{C}$, on fait apparaître le facteur d'amplification : $G(z)=1+z+\frac{z^{2}}{2}$.

Rigoureusement, la condition de stabilité de von Neumann s'écrit $|G(z)| \leq 1+C \Delta t, C>0$ étant une constante donnée. Cette constante quantifie l'amplification d'erreur que l'on peut se permettre. Supposons $u_{n}$

\footnotetext{
${ }^{1}$ La transformée de Fourier d'une fonction $u \in L^{1}(\mathbb{R})$ est notée $\hat{u}(\xi)=\int_{-\infty}^{+\infty} u(x) e^{-i x \xi} d x$. On rappelle que $u \mapsto \frac{1}{\sqrt{2 \pi}} \hat{u}$ est une isométrie de $L^{2}(\mathbb{R})$.
} 
solution de $(E)$ perturbée par $\varepsilon_{n}$, alors par linéarité, $\varepsilon_{n}$ vérifie, après $\frac{T}{\Delta t}$ itérations du schéma en temps :

$$
\left\|\varepsilon_{\frac{T}{\Delta t}}\right\|_{L^{2}} \leq(1+C \Delta t)^{\frac{T}{\Delta t}}\left\|\varepsilon_{0}\right\|_{L^{2}} \leq e^{C T}\left\|\varepsilon_{0}\right\|_{L^{2}}
$$

C'est-à-dire qu'après un temps $T$, cette erreur est au plus multipliée par $e^{C T}$.

Le domaine de stabilité classique d'un schéma, donné par l'ensemble $\{z \in \mathbb{C},|G(z)| \leq 1\}$, ne fait pas intervenir le pas de temps $\Delta t$ et correspond à tous les symboles $\sigma \in \mathbb{C}$ d'opérateurs $F$ pour lesquels $\left\|u_{n+1}\right\|_{L^{2}} \leq\left\|u_{n}\right\|_{L^{2}}$. Par exemple, pour le schéma de Runge-Kutta d'ordre $2(3),|G(i \zeta)|^{2}=1+\frac{\zeta^{4}}{4}>1$ pour $\zeta \in \mathbb{R}^{*}$, donc l'opérateur de convection, de symbole $i \zeta$, ne fait pas partie du domaine de stabilité de (3).

\section{Mise en Évidence De La COndition $\Delta t \leq C \Delta x^{\alpha}$ AVEC $\alpha>1$}

2.1. Stabilité sous $\left\|u_{n+1}\right\|_{L^{2}} \leq(1+C \Delta t)\left\|u_{n}\right\|_{L^{2}}$

Le schéma de Runge-Kutta 2 (3) avec le facteur d'amplification $|G(i \Delta t a \xi)|^{2}=1+\frac{\Delta t^{4} a^{4} \xi^{4}}{4}$ est instable selon le critère usuel $|G| \leq 1$. Maintenant, regardons de plus près ce qui se passe pour $|G| \leq 1+C \Delta t$. Tout d'abord, $\xi$ décrit l'ensemble des fréquences présentes dans la simulation, donc en spectral i.e. avec $u(x)=$ $\sum_{k=-N / 2+1}^{N / 2} c_{k} \exp \left(\frac{2 i \pi k x}{N}\right)$ et $N=\frac{1}{\Delta x}$, on a $\xi \in\left[-\frac{\pi}{\Delta x}, \frac{\pi}{\Delta x}\right]$. Ainsi

$$
|G(\Delta t i a \xi)|^{2} \leq 1+\frac{\Delta t^{4} a^{4} \pi^{4}}{4 \Delta x^{4}}
$$

Cela implique $|G|^{2} \leq 1+2 C \Delta t$ pour $\frac{\Delta t^{4} a^{4} \pi^{4}}{4 \Delta x^{4}} \leq 2 C \Delta t$, d'où la condition de stabilité exacte :

$$
\Delta t \leq 2 C^{\frac{1}{3}}\left(\frac{\Delta x}{\pi a}\right)^{\frac{4}{3}} .
$$

On peut généraliser ce critère à d'autres schémas : étant donné un schéma numérique ayant comme facteur d'amplification :

$$
G(z)=\sum_{\ell=0}^{s} \beta_{\ell} z^{\ell},
$$

on obtient, pour $z=i \zeta, \zeta \in \mathbb{R}$, i.e. l'équation de transport,

$$
|G(z)|^{2}=\sum_{\ell=0}^{s} S_{\ell} \zeta^{2 \ell}
$$

avec, en posant $\beta_{j}=0$ si $j>s$, pour $0 \leq \ell \leq s$,

$$
S_{\ell}=\sum_{j=0}^{2 \ell}(-1)^{\ell+j} \beta_{j} \beta_{2 \ell-j} .
$$

Pour des raisons de consistance $\beta_{0}=\beta_{1}=1$, et donc $S_{0}=1$. Si, par ailleurs $S_{1}=\cdots=S_{p-1}=0$ et $S_{p}>0$, alors $|G(z)|^{2}=1+S_{p} \zeta^{2 p}+o\left(\zeta^{2 p}\right)$ et la condition de stabilité de von Neumann $|G(z)| \leq 1+C \Delta t$ est assurée par :

$$
\Delta t \leq\left(\frac{2 C}{S_{p}}\right)^{\frac{1}{2 p-1}}\left(\frac{\Delta x}{\pi a}\right)^{\frac{2 p}{2 p-1}}
$$

D'où les exposants $\alpha=2, \frac{4}{3}, \frac{6}{5}, \ldots$ pour $p=1,2,3, \ldots$ dans $\Delta t \leq C^{\prime} \Delta x^{\alpha}$. 
Le facteur d'amplification $G$ est intimement lié au graphe de stabilité du schéma numérique (voir Fig. 2 pour des exemples de tracés), comme le montre le résultat suivant :

Théorème 1. Si, près de zéro, le bord du domaine de stabilité $z \in \mathbb{C}$ est tangent à gauche à l'axe de (Oy) :

$$
z=i(\theta+o(\theta))+T_{2 r} \theta^{2 r}+o\left(\theta^{2 r}\right), \quad \text { avec } \quad r \in \mathbb{Z}_{+}^{*} \quad \text { et } T_{2 r}<0, \quad \text { pour } \theta \rightarrow 0,
$$

alors un schéma centré en espace résolvant l'équation de transport doit vérifier la condition de stabilité :

$$
\Delta t \leq\left(\frac{C}{-T_{2 r}}\right)^{\frac{1}{2 r-1}}\left(\frac{\Delta x}{\pi a}\right)^{\frac{2 r}{2 r-1}} .
$$

Ce théorème est démontré dans le cas spectral dans [3]. Comme augmenter l'ordre d'un schéma accroît le degré de tangence de son domaine de stabilité à l'axe $(O y)$ (la réciproque se révélant fausse), on a :

Théorème 2. Un schéma d'ordre $2 r$ (ou $2 r+1$ ) est stable pour l'équation de transport, au pire sous la condition :

$$
\Delta t \leq C^{\prime} \Delta x^{\frac{2 r+2}{2 r+1}} .
$$

Pour la preuve de ce théorème, on pourra se rapporter à la référence [3].

\subsection{Exemples pris dans la littérature}

Ce résultat s'applique à de nombreux schémas en temps utilisés en simulation numérique. Associés à des discrétisations spectrales ou centrées en espace, leur stabilité au sens de von Neumann s'écrit :

- Pour Euler explicite, $\Delta t \leq 2 C\left(\frac{\Delta x}{\pi a}\right)^{2}$,

- Pour Runge-Kutta $2, \Delta t \leq 2 C^{1 / 3}\left(\frac{\Delta x}{\pi a}\right)^{4 / 3}$,

- Pour le schéma d'extrapolation d'ordre 2, très utilisé en mécanique des fluides pour trouver des états stationnaires

$$
\frac{3 u_{n+1}-4 u_{n}+u_{n-1}}{2 \Delta t}=2 F\left(u_{n}\right)-F\left(u_{n-1}\right),
$$

le bord du domaine vérifie $z=-\frac{1-4 e^{i \theta}+3 e^{2 i \theta}}{2-4 e^{i \theta}}=i \theta-\frac{3}{4} \theta^{4}+o(i \theta)+o\left(\theta^{4}\right)$, d'où d'après le théorème 1

$$
\Delta t \leq\left(\frac{4}{3}\right)^{1 / 3} C^{1 / 3}\left(\frac{\Delta x}{\pi a}\right)^{4 / 3}
$$

- Le schéma d'Adams-Bashforth d'ordre 2 s'écrit $u_{n+1}=u_{n}+\frac{3}{2} \Delta t F\left(u_{n}\right)-\frac{1}{2} \Delta t F\left(u_{n-1}\right)$, et le bord de son domaine de stabilité vérifie $z=\frac{e^{i \theta}-1}{\frac{3}{2}-\frac{1}{2} e^{-i \theta}}=i \theta-\frac{1}{4} \theta^{4}+o(i \theta)+o\left(\theta^{4}\right)$. D'où la condition

$$
\Delta t \leq 2^{2 / 3} C^{1 / 3}\left(\frac{\Delta x}{\pi a}\right)^{4 / 3}
$$

- Le schéma nommé Butcher's Lobatto $4[5]$ vérifie $G(z)=\frac{1+3 z / 4+z^{2} / 4+z^{3} / 24}{1-z / 4}$ et

$$
\Delta t \leq 1152^{1 / 5} C^{1 / 5}\left(\frac{\Delta x}{\pi a}\right)^{6 / 5}, \quad 1152^{1 / 5} \sim 4.095 .
$$


- Le schéma de Runge-Kutta d'ordre 5 tiré de [2] a comme facteur d'amplification $G(z)=1+z+\frac{z^{2}}{2}+$ $\frac{z^{3}}{6}+\frac{z^{4}}{24}+\frac{z^{5}}{120}+\frac{z^{6}}{1280}$, ce qui mène à la condition de stabilité :

$$
\Delta t \leq\left(\frac{11520}{7}\right)^{1 / 5} C^{1 / 5}\left(\frac{\Delta x}{\pi a}\right)^{6 / 5}, \quad\left(\frac{11520}{7}\right)^{1 / 5} \sim 4.398 .
$$

- Le schéma de Butcher's Lobatto $6[5]$ satisfait $G(z)=\frac{1+2 z / 3+z^{2} / 5+z^{3} / 30+z^{4} / 360}{1-z / 3+z^{2} / 30}$, et a comme condition de stabilité :

$$
\Delta t \leq 259200^{1 / 7} C^{1 / 7}\left(\frac{\Delta x}{\pi a}\right)^{8 / 7}, \quad 259200^{1 / 7} \sim 5.934 .
$$

- Pour Dormand \& Prince d'ordre $8[6]$, avec $G(z)=\sum_{j=0}^{8} \frac{z^{j}}{j !}+0.27521279901 \cdot 10^{-5} * z^{9}+0.24231996586959$. $10^{-6} * z^{10}+0.24389718205443 \cdot 10^{-7} * z^{11}-0.2034615289686 \cdot 10^{-9} * z^{12}$ on obtient

$$
\Delta t \leq 6.861876 C^{1 / 9}\left(\frac{\Delta x}{\pi a}\right)^{10 / 9}
$$

\subsection{Effets de la discrétisation spatiale}

Ces résultats s'appliquent en fait à de très nombreuses discrétisations. Seul le fait d'utiliser une discrétisation spatiale de type upwind permet d'y contrevenir. Mais même à ce moment-là, une analyse de stabilité de von Neumann du même type que ci-dessus nous permet de tirer des conclusions originales.

Considérons la discrétisation en espace par différences finies : $\partial_{x} u \sim \frac{1}{\Delta x} \sum_{k} \alpha_{k} u^{k}$ avec $\sigma(\xi)=\sum_{k} \alpha_{k} e^{i k \xi} \notin$ $i \mathbb{R}$. Afin que cette discrétisation soit admissible, il faut que son spectre soit localisé sur la partie gauche du plan complexe. Près de l'origine, ce spectre est tangent à l'axe $(O y)$ et vérifie de façon approchée $x=-K y^{2 q}$, avec $K>0$ et $q$ un entier. On rappelle que l'entier $p$, lié au domaine de stabilité du schéma en temps, est donné $\operatorname{par}(12)$.

Une analyse de stabilité de von Neumann $|G| \leq 1+C \Delta t$ nous permet alors de trouver à nouveau des conditions de stabilité du type $\Delta t \leq C \Delta x^{\alpha}$, avec cette fois-ci :

- $\alpha=1$ si $q \leq p$ (stable sous condition CFL linéaire),

- $\left.\left.\alpha=\frac{p(2 q-1)}{(2 p-1) q} \in\right] 1,2\right]$ si $q>p$.

On remarque que pour $q=+\infty$ (schéma aux différences finies centré) on retrouve le cas spectral: $\alpha=\frac{2 p}{2 p-1}$.

\section{TESTS NUMÉRIQUES}

Une manière simple de produire des schémas numériques ayant des facteurs d'amplification donnés est de considérer les schémas suivants :

$$
u_{n+1}=u_{n}+\alpha_{1} \Delta t F\left(u_{n}+\alpha_{2} \Delta t F\left(u_{n}+\alpha_{3} \Delta t F\left(u_{n}+\ldots\right)\right)\right) .
$$

Alors les $\beta_{\ell}$ de (8) et les $\alpha_{\ell}$ sont liés par la relation $\beta_{m}=\prod_{\ell=1}^{m} \alpha_{\ell}$. Ainsi on construit des schémas numériques (d'ordre deux maximum) ayant les facteurs d'amplification voulus, et dont les graphes de stabilité présentent différentes tangences à l'axe $(O y)$ comme le montre la Fig. 2 à gauche. On se rapportera à la référence [3] pour le calcul des coefficients correspondants.

On teste leur stabilité avec l'équation de Burgers $\partial_{t} u+u \partial_{x} u=0$ avec $x \in \mathbb{T}(-1,1), t \in[0,1], u_{0}(x)=$ $10-0.1 \sin (\pi x)$ i.e. dans des conditions proches du transport pur, avec une discrétisation spectral-Fourier en espace. On prend comme critère de divergence : $\left\|u_{N}(\cdot, t)\right\|_{T V}>K\left\|u_{0}(\cdot)\right\|_{T V}$ avec $K=1.1$.

Une expérience numérique plus complexe avec l'équation de Navier-Stokes dans le cas d'une petite viscosité $\nu$, utilisant une discrétisation en espace pseudo-spectral, un schéma de Runge-Kutta 2 en temps (impliquant 


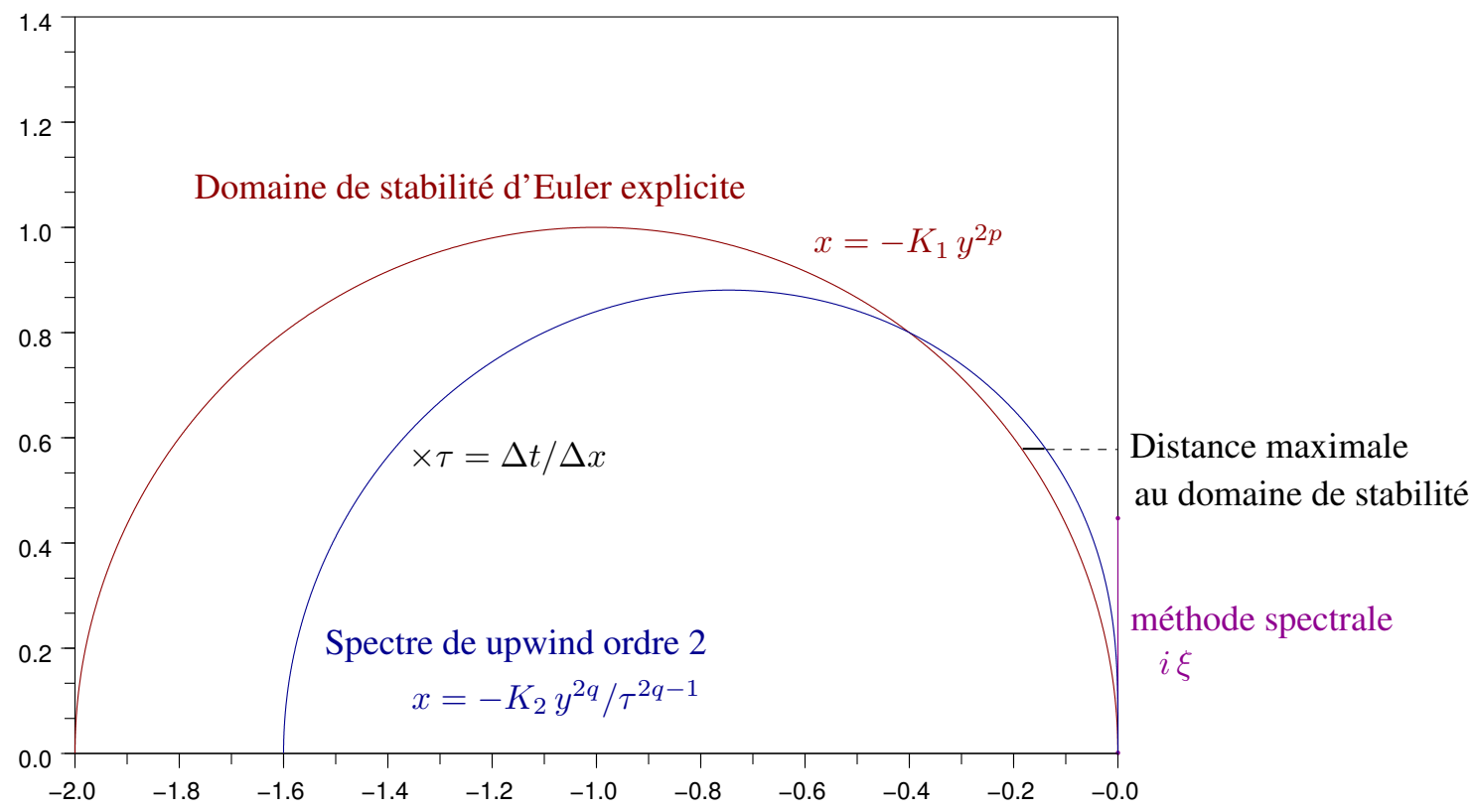

Figure 1. Spectre d'un schéma en espace upwind d'ordre $2(q=2)$ et domaine de stabilité du schéma d'Euler explicite en temps $(p=1)$. Afin d'assurer la stabilité numérique, le spectre ne doit pas trop s'éloigner du domaine de stabilité.
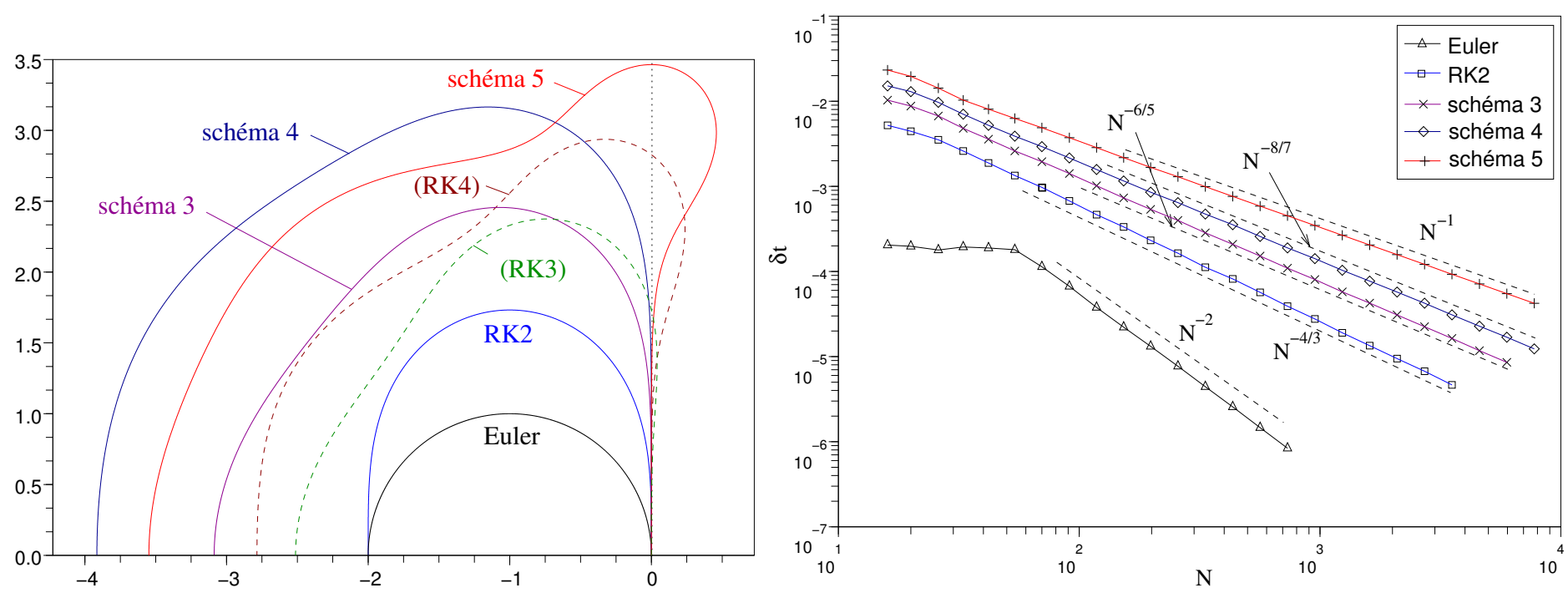

FIguRE 2. À gauche, les domaines de stabilité pour différents schémas en temps : certains classiques, les RK $\ell$ avec $1 \leq \ell \leq 4$ et d'autres créés pour les besoins de cette étude. À droite les pas de temps maximaux correspondant à ces schémas, obtenus expérimentalement en fonction du nombre de points $N=2 / \Delta x$.

une stabilité en $\Delta t \leq C \Delta x^{4 / 3}$ ) et une méthode de pénalisation pour les bords du domaine (avec le paramètre $\eta)$, corrobore cette étude de stabilité, voir Fig. 3. 

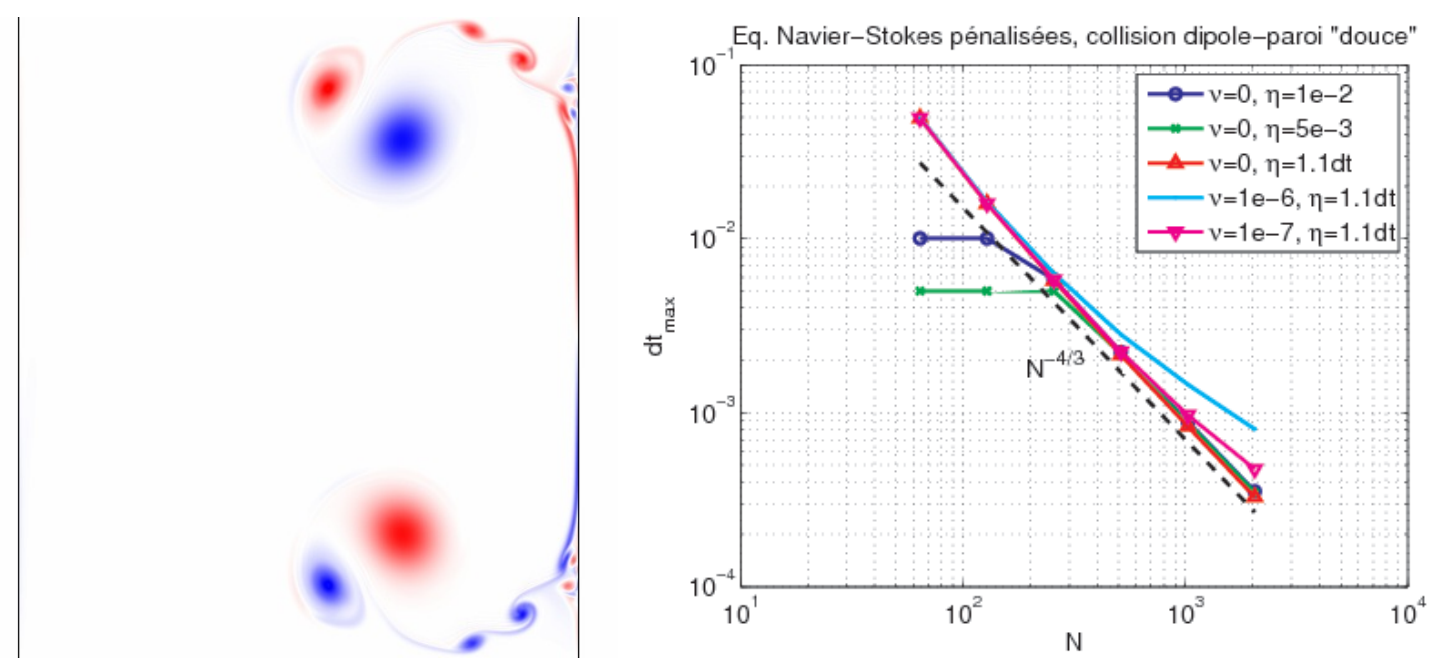

Figure 3. À gauche, une expérience numérique de mécanique des fluides : l'interaction dipôle/paroi. À droite le pas de temps maximum en fonction de $N=2 / \Delta x$ déterminant le nombre de points de grille $N^{2}$.

\section{CONCLUSION}

Ainsi, on comprend bien d'où provient la condition de stabilité $\Delta t \leq \Delta x^{\alpha}$ avec $\alpha>1$ qui se rencontre parfois dans les simulations numériques où domine la convection [9]. Et une simple analyse de stabilité de von Neumann permet d'en rendre compte, à condition de considérer un accroissement de l'erreur en $C \Delta t$ à chaque pas de temps.

Reste à savoir pourquoi des schémas a priori défavorables continuent à être utilisés pour faire des simulations de turbulence. Outre le fait qu'un exposant $\frac{4}{3}$ passe plus inaperçu qu'un exposant 2 , un élément de réponse se trouve dans l'amortissement des petites échelles induit par les schémas stables sous condition CFL.

En effet, considérons le facteur d'amplification $|G(i \Delta t a \xi)|^{2}=1+S_{p} a^{2 p}(\xi \Delta t)^{2 p}+o\left((\xi \Delta t)^{2 p}\right)$ avec $S_{p}<0$. Il correspond à une évolution spectrale $|\widehat{u}(T, \xi)|^{2} \approx e^{S_{p} \xi^{2 p} a^{2 p} \Delta t^{2 p-1} T}\left|\widehat{u_{0}}(\xi)\right|^{2}$. Ainsi, si on souhaite conserver toutes les échelles, c'est-à-dire $e^{S_{p} \xi^{2 p} a^{2 p} \Delta t^{2 p-1} T} \geq K$ avec $0<K<1$ proche de $1, \forall \xi \in\left[0, \frac{1}{\Delta x}\right]$, on obtient la condition suivante :

$$
\Delta t \leq\left(\frac{\log K}{S_{2 p} T}\right)^{\frac{1}{2 p-1}}\left(\frac{\Delta x}{\pi a}\right)^{\frac{2 p}{2 p-1}} .
$$

c'est-à-dire le même type de conditions que pour les schémas instables sous condition CFL linéaire.

Par exemple, le schéma de Runge-Kutta 3 a comme facteur d'amplification $G(z)=1+z+\frac{z^{2}}{2}+\frac{z^{3}}{6}$, donc $p=2$ et $S_{2}=-\frac{1}{12}<0$. Ce schéma est stable sous condition CFL linéaire. Mais, pour conserver toutes les échelles, le pas de temps doit vérifier la condition $\Delta t \leq 12^{1 / 3} C^{1 / 3}\left(\frac{\Delta x}{\pi a}\right)^{4 / 3}$, avec la constante $C=-\frac{2 \log K}{T}$. Cette condition, similaire à la condition de stabilité pour Runge-Kutta 2 peut passer totalement inaperçue alors que l'utilisation de la condition CFL linéaire est susceptible de fausser la cascade turbulente.

\section{REFERENCES}

[1] R. Courant, K. Friedrichs, H. Lewy, On the Partial Difference Equations of Mathematical Physics, IBM Journal, March 1967, translation from a paper originally appeared in Mathematische Annalen 100 32-74, 1928.

[2] M. Crouzeix, A. L. Mignot, Analyse numérique des équations différentielles, Masson, 1992.

[3] E. Deriaz, Stability conditions for the numerical solution of convection-dominated problems with skew-symmetric discretizations, à paraître dans SIAM J. Numer. Anal., 2012. 
[4] E. Deriaz And V. Perrier, Direct Numerical Simulation of turbulence using divergence-free wavelets, SIAM Multiscale Modeling and Simulation 7(3) 1101-1129, 2008.

[5] E. Hairer, S. P. Nørsett, G. Wanner, Solving Ordinary Differential Equations I. Nonstiff Problems. Springer Series in Comput. Mathematics, Vol. 8, Springer-Verlag 1987, Second revised edition 1993.

[6] E. Hairer, G. Wanner, Solving Ordinary Differential Equations II. Stiff and Differential-Algebraic Problems. Springer Series in Comput. Mathematics, Vol. 14, Springer-Verlag 1991, Second revised edition 1996.

[7] R.W.C.P. Verstappen, A.E.P. Veldman, Symmetry-preserving discretization of turbulent flow, J. Comp. Phys. 187 (2003) $343-368$.

[8] P. Wesseling, Von Neumann stability conditions for the convection-diffusion equation, IMA Journal of Numerical Analysis 16:583-598, 1996.

[9] Q. Zhang And C.-W. Shu, Error Estimates to Smooth Solutions of Runge-Kutta Discontinuous Galerkin Methods for Scalar Conservation Laws, SIAM J. Numer. Anal. 42(2): 641-666, 2004. 